\title{
Orthopaedic Trainees Retain Knowledge After a Partner Abuse Course: An Education Study
}

\author{
Kim Madden MSc, Sheila Sprague PhD, \\ Brad A. Petrisor MD, MSc, FRCSC, Forough Farrokhyar PhD, \\ Michelle A. Ghert MD, FRCSC, Marium Kirmani BSc (Cand), \\ Mohit Bhandari MD, PhD, FRCSC
}

Received: 30 September 2014/ Accepted: 17 April 2015/Published online: 28 April 2015

(C) The Association of Bone and Joint Surgeons (B) 2015

\begin{abstract}
Introduction Intimate partner violence (IPV) is a serious global issue that results in a large number of injuries and deaths among women. Educating clinicians about IPV can help providers identify, prevent, and treat victims, and, ultimately, improve care for victims of abuse. We sought to determine the effect of a half-day educational course on IPV for orthopaedic surgical trainees on knowledge and attitudes.

Questions/purposes We asked (1) whether a half-day educational course on IPV can improve orthopaedic surgical trainees' knowledge and (2) attitudes regarding IPV; and (3) whether a course on IPV can be accepted and viewed as valuable by trainees?

Methods Using published research on IPV in patients with musculoskeletal injuries, we developed a half-day educational course. The curriculum included lectures and discussion regarding the basics of IPV, the current state of IPV research, what to do when a patient is a victim or perpetrator, and the orthopaedic surgeon's role in recognizing, preventing, and assisting with IPV. All 33 course
\end{abstract}

The institution of the authors has received, during the study period, funding from the Canadian Institutes of Health Research (CIHR) (Ottawa, Canada) Knowledge Dissemination Grant. Each author certifies that he or she, or a member of his or her immediate family, has no funding or commercial associations (eg, consultancies, stock ownership, equity interest, patent/licensing arrangements, etc.) that might pose a conflict of interest in connection with the submitted article.

All ICMJE Conflict of Interest Forms for authors and Clinical Orthopaedics and Related Research ${ }^{\circledR}$ editors and board members are on file with the publication and can be viewed on request.

Each author certifies that his or her institution approved or waived approval for the human protocol for this investigation and that all investigations were conducted in conformity with ethical principles of research. participants (30 men and three women), all orthopaedic surgical trainees, completed a questionnaire that included general true or false or agree or disagree statements regarding their knowledge, attitudes, and practices of IPV in the musculoskeletal setting; the questionnaire also included a knowledge test of 25 true or false statements. The questionnaire was administered immediately before, immediately after, and 3 months after the course; $76 \%$ (25 of 33 ) took the test immediately after the course and $82 \%$ (27 of 33) completed the test at 3 months. Participant knowledge scores were compared across the three different times to determine the effect of the course.

Results Participants increased their knowledge after the course, and the increased knowledge was retained at retesting at 3 months; the mean percentage of correct answers before the course was $57 \%$, which increased to $73 \%$ after the course, and was $68 \% 3$ months later $(\mathrm{F}=9.505$; $\mathrm{p}=0.001)$. Before the course, most of the course participants (30 of 32; 94\%) agreed that IPV is an important issue; agreement increased to $100 \%$ immediately after the course. The largest change in attitude was in response to

Electronic supplementary material The online version of this article (doi:10.1007/s11999-015-4325-7) contains supplementary material, which is available to authorized users.

K. Madden ( $₫)$, S. Sprague, F. Farrokhyar, M. Bhandari Department of Clinical Epidemiology \& Biostatistics, McMaster University, 293 Wellington St. North, Suite 110, Hamilton, ON L8L 8E7, Canada

e-mail: maddenk@mcmaster.ca

S. Sprague, B. A. Petrisor, F. Farrokhyar,

M. A. Ghert, M. Kirmani, M. Bhandari

Department of Surgery, McMaster University, Hamilton,

ON, Canada 
the statement: "I am skeptical that the health care system has the resources to screen for IPV." Before the course, $53 \%$ (17 of 32) of trainees endorsed this statement, but the percent decreased to $36 \%$ (nine of 25) after the course and remained low at $33 \%$ (nine of 27), at the 3 -month test.

Conclusions Our findings show that a short course on IPV in patients with musculoskeletal injuries led to an improvement and retention of knowledge 3 months after the course. Based on our findings, we recommend that IPV education be integrated in training programs for orthopaedic surgeons. Future projects should focus on developing and implementing a sustainable education program that can affect practice for healthcare professionals and trainees in multiple clinical settings.

\section{Introduction}

Intimate Partner Violence (IPV) is a serious global issue that results in a large number of injuries and deaths among women [10]. A recent systematic review of 37 IPV prevalence studies reported the lifetime prevalence of IPV seen by practitioners in emergency and family medicine practices is $40 \%$ and $38 \%$, respectively [15]. Fifteen percent of IPV-related injuries are serious enough to warrant medical attention [4]. The injuries often require the consultation of orthopaedic surgeons. Bhandari et al. [2] reported that sprains, dislocations, fractures, and foot injuries accounted for $28 \%$ of all physical manifestations of IPV among women who were identified in a 2 -year period by a domestic abuse community program. However, the number of IPV cases known by orthopaedic surgeons in Canada may be underestimated; $87 \%$ of orthopaedic surgeons who participated in a Canada-wide study believed that women victims of IPV accounted for less than $1 \%$ of patients in their care [4]. Moreover, 9\% of the respondents believed that inquiring about IPV was an invasion of the patient's privacy, and $11 \%$ believed that ruling out IPV as the cause of injury was not part of their duty [4]. This may be because clinicians in disciplines such as family medicine and emergency medicine focused their attention on the issue in the past; however, orthopaedic surgeons have just begun discussing IPV in clinical practice [3, 8, 19].

There are many barriers that surgeons face when they suspect a patient is a victim of IPV [17, 18]. However, research suggests that many of these challenges can be overcome with increased knowledge of IPV in a clinical setting [17-19]. Unpublished meta-analysis data from our group shows that IPV education programs for healthcare professionals significantly increased knowledge and screening behaviors of healthcare professionals compared with those without the training in seven randomized studies. An additional 25 nonrandomized studies of 27 studies concluded that IPV educational programs significantly increased knowledge and skills regarding screening patients for IPV. However, none of the studies was conducted in the field of orthopaedic surgery. We therefore developed a course aimed at educating orthopaedic surgeons and other healthcare professionals regarding how to support women who have experienced IPV.

Our prospective study aimed to investigate to what extent a half-day course could change the knowledge and attitudes of orthopaedic trainees about IPV in the musculoskeletal injury setting immediately after the course and 3 months after the course. We asked (1) whether a half-day educational course on IPV improved orthopaedic surgical trainees' knowledge and (2) attitudes about IPV, and (3) whether a course on IPV is accepted and viewed as valuable by trainees?

\section{Methods}

We conducted a prospective study wherein orthopaedic trainees (fellows, residents, and medical students) completed a self-reported written questionnaire before attending a half-day course on IPV, again immediately after the course, and then once more 3 months after the course. The medical students were senior students who attended orthopaedic rounds because they are interested in a career in orthopaedics. We chose to retain the medical students in the sample of orthopedic trainees because they are currently learning about orthopaedics.

We received approval from the Hamilton Integrated Research Ethics Board (Project 12-339) before conducting this study. We did not seek written consent from participants for our study as submission of questionnaires was considered implied consent. We kept all test scores strictly confidential and removed identifiers as soon as possible after the test. The coding list was available to only the first author (KM).

Our study had minimal inclusion and exclusion criteria. To be eligible, participants had to attend the half-day course, be an orthopaedic surgery trainee (including orthopaedic surgery fellows, orthopaedic surgery residents, and medical students on an orthopaedics elective), and be able to understand, read, and write in English. Participants were excluded if they were unwilling to complete the study questionnaires. Orthopaedic surgery trainees at McMaster University were required to attend the course as part of their training but were told that they could decline participation in the study without penalty. We did not invite practicing orthopaedists to participate. Training practicing surgeons is important, however, a different type of course (perhaps with Continuing Medical Education credits) will need to be developed. Trainees, however, have mandatory sessions that they must attend as part of their training and 
therefore are a "captive audience". Future studies may focus on a wider orthopaedic audience in a Continuing Medical Education format, but the scope of our study was trainees only.

The course was developed with consultation from IPV researchers, social workers, clinical psychologists, research methodologists, surgeon-educators, orthopaedic surgeons, and the study authors performing an in-depth literature review on IPV education. The course consisted of seven modules, all of which were tailored to orthopaedic surgery trainees (Fig. 1).

The objective of Module 1 was to learn the definitions of IPV and the effect of IPV in society. In Module 2, the objective was to learn about case presentations of IPV in a trauma setting. Module 3 provided an overview of the research conducted to date on IPV in orthopaedic surgery. In Module 4, a leading IPV researcher presented a model for practice that highlighted important recommendations for implementing a system to help surgeons recognize victims of IPV. Learning objectives for Module 5 included the steps a surgeon could take if they suspected their patient to be a victim of IPV. Learning objectives for Module 6 included what to do if the patient was a suspected perpetrator. The course ended with Module 7, which aimed to teach course participants about the Canadian Orthopaedic Association's position on IPV, emphasizing how surgeons can help victims of IPV and that surgeons are encouraged to educate themselves about IPV [19]. Course materials are available on request from the corresponding author (KM).

The questionnaire chosen for our study was a selfreported written questionnaire developed by the current authors. We were unable to find an existing knowledge test that specifically tested basic knowledge of IPV as it relates to orthopaedic surgery and physical injury; therefore, we developed a knowledge test in collaboration with orthopaedic surgeons, methodologic experts, IPV researchers, and social workers. Although the questionnaire is not formally validated, we pilot tested the questionnaire on five nonphysician orthopaedic research assistants and one orthopaedic resident to improve the clarity of the information offered. We used nonphysician research assistants for convenience to finesse wording and clarity. After pilot testing, we removed ambiguous true or false statements and refined some wording based on feedback from the research assistants and resident.

The knowledge test and course content were developed independently with the intention of testing the participants' general knowledge of IPV, not the ability to recall what was presented during the course. The knowledge test consisted of 25 statements and included the following response options: (1) True; (2) False; and (3) Unsure. Course participants completed the same questionnaire at each of the three study times. (Appendix 1. Supplemental material is available with the online version of $\operatorname{CORR}^{\circledR}$ ). Only the answers from the IPV knowledge section were
Fig. 1 The course modules and learning objectives are shown. Course objectives include definitions and impact of IPV, case presentations in trauma, IPV research specific to orthopaedics, a surgeon's role, how to assist victims and perpetrators, and the Canadian Orthopaedic Association's position on IPV. IPV= intimate partner violence.

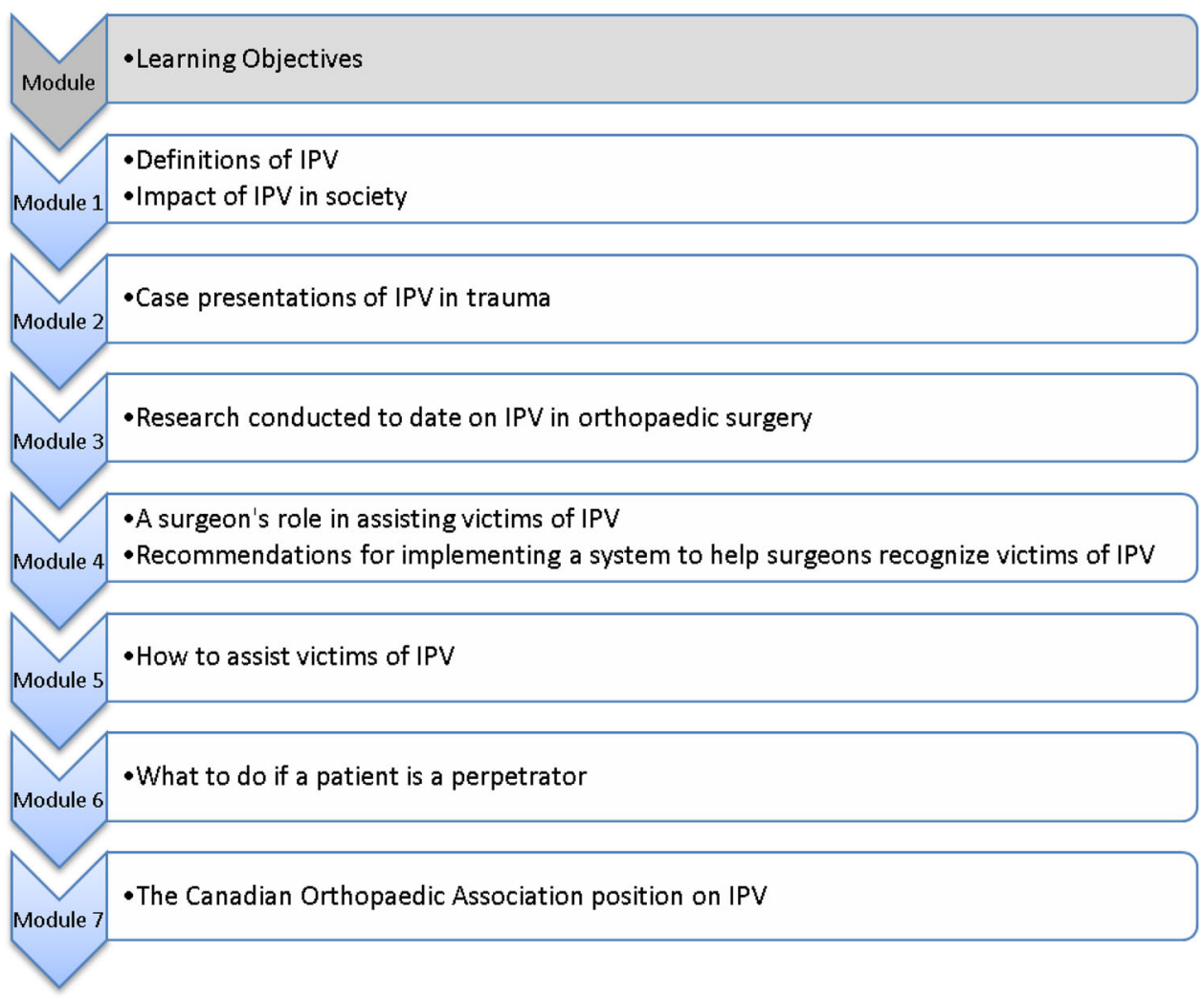




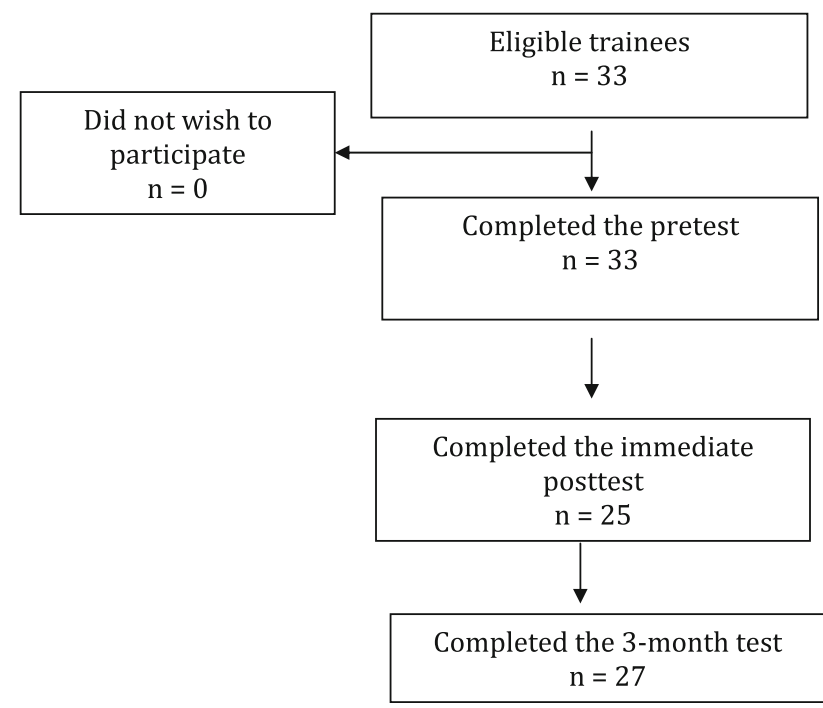

Fig. 2 The flow diagram of our study shows that 33 trainees were eligible to participate. No trainees declined participation. Twenty-five trainees completed the posttest and 27 completed the 3 -month test.

used to determine the primary outcome of the study. The maximum achievable score was 25 and the minimum was 0 . Responses of "unsure" were counted as incorrect answers. The rationale for including "unsure" as a response option was to limit guessing the correct answer.

The pretest questionnaire also included demographic questions, including participant race, sex, age, and whether the participant had taken a previous course in IPV. The posttest questionnaire collected some feedback information regarding course format, satisfaction with the learning objectives, and audience interest. The 3-month questionnaire had additional questions regarding how the course had affected the clinicians' practices.

The first questionnaire was administered on site at our institution before the start of the classroom session. Before distributing the questionnaire, the protocol, risks, and benefits of the study were discussed with the participants and they were given the opportunity to ask questions about the study. The second questionnaire also was administered onsite immediately after the course. The third questionnaire was sent to participants by e-mail 3 months after the date of the course. The e-mail provided a link to a secure website where the questionnaire could be answered confidentially. Research personnel followed up with course participants to ensure completion of the questionnaire up to four times for each time, and again in person at rounds for the 3-month questionnaire.

Thirty-three orthopaedic trainees attended the course and completed the pretest. Twenty-five course participants completed the immediate posttest, and 27 completed the 3-month test. The study flow diagram (Fig. 2) illustrates the change in participant numbers through the progress of the study. Eight residents needed to leave the session
Table 1. Participant demographics $(n=33)$

\begin{tabular}{lc}
\hline $\begin{array}{l}\text { Demographic } \\
\text { Age (years, mean } \pm \text { SD) }\end{array}$ & $30 \pm 5$ \\
\hline Sex (n, \%) & $30(91)$ \\
Men & $3(9)$ \\
Women & \\
Ethnicity/race (n, \%) & $15(46)$ \\
White & $7(21)$ \\
South Asian & $5(15)$ \\
Middle Eastern & $5(15)$ \\
Asian & $1(3)$ \\
Native Canadian & \\
Occupation (n, \%) & $29(88)$ \\
Surgical resident & $2(6)$ \\
Surgical fellow & $2(6)$ \\
Medical student & \\
Previous IPV training (n, \%) & $18(55)$ \\
None & $15(45)$ \\
Some & $0(0)$ \\
Extensive & \\
\hline
\end{tabular}

$\mathrm{IPV}=$ intimate partner violence.

before the immediate posttest was completed owing to clinical duties. We made every attempt to contact these individuals for the 3-month test.

The study participants consisted mainly of surgical residents (88\%), most of whom were men (91\%); approximately $1 / 2$ were white $(45 \%)$ and had a mean age of 30 years (SD, 5 years) (Table 1$)$.

\section{Data Analysis}

Demographic data were analyzed descriptively. Continuous data were reported as means and SD, and categorical data by frequency counts and proportions. The comparative data were analyzed using one-way repeated ANOVA to compare the differences among the group means of the three tests and we performed a Bonferroni post hoc test. "Unsure" responses were considered to be incorrect. All analyses were completed using SPSS ${ }^{\circledR}$ version 20 (IBM, Armonk, NY, USA). For comparative analyses, p less than 0.05 was considered statistically significant.

\section{Results}

Participants increased their knowledge regarding IPV after the course and the increased knowledge was retained at the 3-month test (Fig. 3). The mean percentage of correct responses before the course was $57 \%$. The mean 
percentage of correct responses immediately after the course increased from $57 \%$ to $73 \%$. $(p=0.001)$. Three months after the course, the mean percentage of correct scores decreased slightly from the immediate postcourse scores but still increased by more than $10 \%$ (95\% CI, 1.4-19; Table 2) and remained higher than baseline scores at $68 \%(95 \% \mathrm{CI}, 62-73 ; \mathrm{p}=0.018)$. There was no decrease in mean correct responses from immediate postcourse scores to 3 -month test scores $(5 \%$; $95 \% \mathrm{CI},-5$ to $4 ; \mathrm{p}=0.530$ ), indicating that the results did not decrease 3 months after the course. The greatest changes in scores (proportion of trainees answering the question correctly) were seen in the following statements: (1) Head and neck injuries are the most common physical manifestations of IPV (increased from $21 \%$ to $96 \%$; $\mathrm{p}<0.001$ ); (2) Musculoskeletal injuries are the most prevalent type of IPV injury (increased from $33 \%$ to $76 \%$; $p=0.001$ ); (3) There is a lack of IPV screening tools that can be used in a clinical setting (increased from $18 \%$ to $42 \% ; \mathrm{p}=0.008$ ); and (4) The majority of abused women support routine screening for IPV (increased from $42 \%$ to $83 ; p=0.004$ ).

The participants' baseline responses on knowledge and attitudes were favorable. For example, $93 \%$ of participants agreed that IPV was an important issue before the course, which increased to $100 \%$ immediately after the course (Table 3). The largest change in attitude was in response to the statement: "I am skeptical that the healthcare system

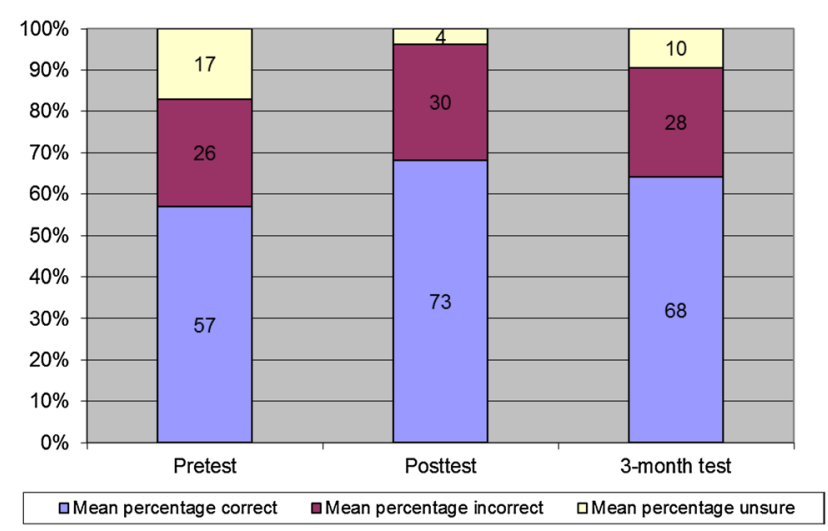

Fig. 3 Knowledge test scores show that the mean percentage of correct scores increased after the course and remained nearly as high 3 months later. has the resources to screen for IPV." Fifty-three percent of trainees endorsed this statement before the course, but the response percentage decreased to $36 \%$ after the course, and remained low at $33 \%$ at the 3 -month test. Conversely, before the course, only $7 \%$ of trainees thought that asking about IPV was dangerous for the patient, but after the course, participant response increased to $32 \%$ and remained higher than baseline response 3 months later $(15 \%)$. Of the trainees, $34 \%$ agreed that they would not know what to do if their patient disclosed IPV before the course. This changed to only $8 \%$ of trainees not knowing what to do after the course, but the attitude was not retained 3 months later (26\% would not know what to do at 3 months; Table 3). The attitude information is reported descriptively only. No comparative statistics were performed because of small values in many cells (ie, many cells contain 0 ) not conducive to chi-square testing.

The training course was acceptable to participating surgical trainees. According to the answers given in the postcourse questionnaire, all the participants agreed, and of those 54\% strongly agreed that the course was informative and interesting. Ninety-six percent of the participants also indicated that they would recommend the course to their colleagues.

\section{Discussion}

Several studies have shown that IPV is underreported among women who seek medical attention [7, 13, 22]. Orthopaedic injuries make up approximately $28 \%$ of the physical manifestations of IPV [2], however, IPV still is underemphasized in clinical medicine and surgery. This underreporting and lack of emphasis on IPV has been hypothesized to be highly detrimental to outcomes, as $44 \%$ of victims of domestic violence-related homicide had presented to an emergency department within 2 years before their death [13]. Of the patients who presented to an emergency department, domestic violence was documented in medical records in only two cases and there was no intervention noted [13]. This indicates that there is still work to be done to improve reporting and intervention for victims of abuse. Our course was designed to provide a pragmatic approach to remedy the gap of IPV knowledge

Table 2. Results from Bonferroni post hoc test

\begin{tabular}{llll}
\hline Comparison times & Mean difference in scores & $95 \%$ CI for mean difference & $p$ Value \\
\hline Before course to immediately after course & $16 \%$ (from $57 \%$ to $73 \%$ ) & $7 \%-25 \%$ & $0.001 *$ \\
Before course to 3 months after course & $11 \%$ (from 57\% to 68\%) & $1 \%-19 \%$ & $0.018 *$ \\
Immediately after course to 3 months after course & $-5 \%$ (from 73\% to 68\%) & $-15 \%$ to $4 \%$ & 0.530 \\
\hline
\end{tabular}

* Mean difference is significant at $\mathrm{p}<0.05$. 
Table 3. Participants' perceived knowledge and attitudes

\begin{tabular}{|c|c|c|c|}
\hline Statement & $\begin{array}{l}\text { Before course } \\
\text { Number }(\%)\end{array}$ & $\begin{array}{l}\text { Immediately after course } \\
\text { Number }(\%)\end{array}$ & $\begin{array}{l}3 \text { months after course } \\
\text { Number }(\%)\end{array}$ \\
\hline \multicolumn{4}{|c|}{ IPV is a serious issue } \\
\hline Disagree & $0(0)$ & $0(0)$ & $4(15)$ \\
\hline Unsure & $2(6)$ & $0(0)$ & $0(0)$ \\
\hline Agree & $30(94)$ & $25(100)$ & $23(85)$ \\
\hline \multicolumn{4}{|c|}{ IPV is a private issue that should be settled only by the couple involved. } \\
\hline Disagree & $30(94)$ & $25(100)$ & $26(96)$ \\
\hline Unsure & $1(3)$ & $0(0)$ & $0(0)$ \\
\hline Agree & $1(3)$ & $0(0)$ & $1(4)$ \\
\hline \multicolumn{4}{|c|}{ It is important for healthcare professionals in trauma settings to talk to patients about IPV. } \\
\hline Disagree & $1(3)$ & $0(0)$ & $0(0)$ \\
\hline Unsure & $2(6)$ & $0(0)$ & $1(4)$ \\
\hline Agree & $29(91)$ & $25(100)$ & $26(96)$ \\
\hline \multicolumn{4}{|c|}{ I am skeptical that the healthcare system has the resources to screen for IPV. } \\
\hline Disagree & $10(31)$ & $16(64)$ & $14(52)$ \\
\hline Unsure & $5(16)$ & $0(0)$ & $4(15)$ \\
\hline Agree & $17(53)$ & $9(36)$ & $9(33)$ \\
\hline \multicolumn{4}{|c|}{ If a healthcare professional asks a patient about IPV it will put the patient in more danger. } \\
\hline Disagree & $25(81)$ & $14(56)$ & $20(74)$ \\
\hline Unsure & $4(13)$ & $3(12)$ & $3(11)$ \\
\hline Agree & $2(6)$ & $8(32)$ & $4(15)$ \\
\hline \multicolumn{4}{|c|}{ I do not know how to screen for IPV. } \\
\hline Disagree & $10(31)$ & $23(92)$ & $20(74)$ \\
\hline Unsure & $5(16)$ & $0(0)$ & $0(0)$ \\
\hline Agree & $17(53$ & $2(8)$ & $7(26)$ \\
\hline \multicolumn{4}{|c|}{ If a patient told me she was a victim of IPV, I would not know what to do. } \\
\hline Disagree & $18(56)$ & $23(92)$ & $20(74)$ \\
\hline Unsure & $3(9)$ & $0(0)$ & $0(0)$ \\
\hline Agree & $11(35)$ & $2(8)$ & $7(26)$ \\
\hline \multicolumn{4}{|c|}{ Most patients would not mind if a healthcare professional asked them about IPV. } \\
\hline Disagree & $5(16)$ & $2(8)$ & $6(22)$ \\
\hline Unsure & $9(29)$ & $5(20)$ & $1(4)$ \\
\hline Agree & $17(55)$ & $18(72)$ & $20(74)$ \\
\hline
\end{tabular}

$\mathrm{IPV}=$ intimate partner violence.

in orthopaedic surgery clinicians $[16,17]$, and in the current study, we sought to evaluate whether the course effectively filled this gap and to ascertain whether a short IPV course can increase knowledge of IPV among surgical trainees. Our findings suggest that the course increased trainees' knowledge regarding IPV and that they retained this knowledge after 3 months.

Limitations of our study include the small sample size and that it was single center. Future studies should be larger, target more orthopaedic training programs, and should be extended to include practicing orthopaedic surgeons. The trainees' high level of knowledge at baseline was another limitation. The study was conducted in a population aware of IPV, as much IPV research has been conducted at McMaster University. The effectiveness of the course may be different in populations that have had less exposure to IPV content. Most of our sample was male participants which is typical of orthopaedic residency programs. The course might have different effectiveness for female participants, but we were unable to explore the effects of gender in this study. Owing to the small sample size, we were unable to analyze data by type of course participant. Medical students may have had additional exposure to this topic before their rotation. Our course also was limited by including only a single didactic lecture component. For more successful uptake of knowledge, 
future courses should include multiple different components, like interactive role-playing, and occur more than once. We were unable to reach eight participants (24\%) immediately after the course, primarily because they needed to leave early to complete clinical duties. The eight participants who left early and did not complete the immediate posttest could have differed from those who did complete the test; for example, they might have been less interested in the topic than their colleagues. We attempted to reduce loss to followup by contacting the participants multiple times by e-mail, by phone, and while they were in clinic or grand rounds, if necessary. We also offered a choice of paper or electronic surveys for convenience. Although improved knowledge can be a useful starting point, it may not translate to clinical practice. Future studies should focus on measuring changes in practice, behavior, knowledge, and attitudes, and the effect of these changes on patients' quality of life.

We found that our short course on IPV improved the knowledge of orthopaedic trainees and that this knowledge was retained at 3 months. To address the low rates of IPV screening among clinicians, previous studies have explored barriers to IPV screening among various healthcare professionals, including emergency department healthcare workers, obstetricians and gynecologists, family physicians, internists, and healthcare staff in family planning organizations $[5,9,20]$. The lack of knowledge and training may be an important factor in the low rates of screening for IPV [6]. Connor et al. [6] conducted a study among dental students and measured knowledge, attitudes, beliefs, and self-reported behaviors regarding IPV. Their study indicated that a sizeable number of students received no IPV training before or during dental school, leading to perceptions that they lack knowledge about IPV and are not well prepared to address the problem of IPV among their patients [6]. Similarly, Wathen et al. [21] conducted a survey of students in 222 programs in dentistry, medicine, nursing, and other allied health professions at the undergraduate and graduate levels and found that only $43 \%$ of undergraduate medical programs and $46 \%$ of undergraduate dentistry programs offered some kind of IPV content; and postgraduate programs ranged from no IPV content (such as in dentistry) to approximately $41 \%$ (nursing). A survey of medical students and surgical residents by Sprague et al. [16] found that there was a general opinion that IPV knowledge was lacking. Park et al. [14], in a study with 982 final-year residents, reported that only $21 \%$ of the residents felt ready to discuss IPV with their patients. However, comparatively, $62 \%$ felt comfortable discussing smoking and 53\% felt prepared to talk about diet and exercise [14]. Furthermore, a report by the World Health Organization shows the imperative need to have a component of violence education in clinical training [23].
Attitudes regarding IPV generally were favorable at baseline. Some attitudes improved, although some did not change or were not retained at the 3-month test. For example, almost all participants stated immediately after the course that they would know what to do if a patient disclosed that they were a victim of IPV, but this rate decreased to $18 \%$ after 3 months. This indicates that orthopaedic surgery trainees could benefit from reinforcement of the concepts introduced in this course. We believe that IPV training should be held several times throughout a trainee's training period to reinforce knowledge and attitudes. Additionally, trainees may benefit from multifocal knowledge and attitude reinforcement using more than just in-class lectures. For example, research suggests that posters and brochures in clinics [11, 12], clinical mentors who routinely ask about IPV [18], and mobile phone or tablet applications with critical resources [1] may be helpful to aid healthcare professionals in routinely asking about IPV. Similar training also should be provided for faculty and practicing orthopaedic surgeons.

The participants generally were supportive of the course. Most would recommend the course to their peers. This finding is supported by a study that showed that medical students and surgical residents are interested in learning about IPV [16]. Future research could focus on the most effective way to deliver such a course so that learning is maximized and participants remain engaged and interested.

To our knowledge, our study is the first educational curriculum-based intervention study focusing on orthopaedic healthcare professionals, and it has the potential to pave the way for large-scale studies. Based on the evidence provided by our study, a course in IPV education can improve surgeon knowledge, which is sustained up to 3 months after taking the course. Information regarding the course is available from the authors on request. Given the high frequency of IPV, this knowledge can aid surgeons in recognizing and thereby working toward reducing the prevalence of IPV. We believe that if knowledge of IPV is integrated as part of a medical curriculum for orthopaedic surgeons, it can help assist abused women to connect with the resources they need. We recommend that education regarding IPV be incorporated in medical school education and continuing medical education programs, and the content could be incorporated in the Accreditation Council for Graduate Medical Education Core Curriculum and become examinable content for American Board of Orthopaedic Surgery exams. Future projects should focus on developing and implementing a sustainable and effective education program for healthcare professionals and trainees in multiple hospitals and academic centers. With the correct knowledge on how to support victims of IPV, surgeons and other physicians can start an open discussion about IPV and help to decrease the frequency of IPV. 
Acknowledgments We thank the course faculty for participation in course development and presentation. Course faculty included Clare Freeman MSW RSW (Interval House Hamilton, Hamilton, Ontario, Canada), Diana Tikasz MSW RSW (Hamilton Health Sciences, Domestic Violence/Sexual Assault Care Centre, Hamilton, Ontario, Canada), Dave Mathews PsyD LICSW (One T Counseling and Consultation Services, Saint Paul, MN, USA), and Emil Schemitsch MD (St. Michael's Hospital, Department of Orthopaedic Surgery, Toronto, Ontario, Canada). We thank Nicole Simunovic MSc (McMaster University, Department of Clinical Epidemiology \& Biostatistics) for assistance with course preparation and Stephanie Noronha BSc (McMaster University, Department of Life Sciences) for assistance with survey administration. We thank Diane HeelsAnsdell MSc (McMaster University, Department of Clinical Epidemiology \& Biostatistics) for statistical assistance. We also thank the course participants for participating in the survey.

\section{References}

1. Balas EA, Weingarten S, Garb CT, Blumenthal D, Boren SA, Brown GD. Improving preventive care by prompting physicians. Arch Intern Med. 2000;160:301-308.

2. Bhandari M, Dosanjh S, Tornetta P 3rd, Matthews D; Violence Against Women Health Research Collaborative. Musculoskeletal manifestations of physical abuse after intimate partner violence. $J$ Trauma. 2006;61:1473-1479.

3. Bhandari M, Sprague S, Dosanjh S, Petrisor B, Resendes S, Madden K, Schemitsch EH; P.R.A.I.S.E. Investigators. The prevalence of intimate partner violence across orthopaedic fracture clinics in Ontario. J Bone Joint Surg Am. 2011;93:132-141.

4. Bhandari M, Sprague S, Tornetta P 3rd, D'Aurora V, Schemitsch E, Shearer H, Brink O, Mathews D, Dosanjh S; Violence Against Women Health Research Collaborative. (Mis)perceptions about intimate partner violence in women presenting for orthopaedic care: a survey of Canadian orthopaedic surgeons. J Bone Joint Surg Am. 2008;90:1590-1597.

5. Colarossi L, Breitbart V, Betancourt G. Barriers to screening for intimate partner violence: a mixed-methods study of providers in family planning clinics. Perspect Sex Reprod Health. 2010;42:236-243.

6. Connor PD, Nouer SS, Mackey SN, Banet MS, Tipton NG. Dental students and intimate partner violence: Measuring knowledge and experience to institute curricular change. $J$ Dent Educ. 2011;75:1010-1019.

7. Dearwater SR, Coben JH, Campbell JC, Nah G, Glass N, McLoughlin E, Bekemeir B. Prevalence of intimate partner abuse in women treated at community hospital emergency departments. JAMA. 1998;280:433-438.

8. Della Rocca G. Editor's spotlight/take 5: Orthopaedic surgeons' knowledge and misconceptions in the identification of intimate partner violence against women. (DOI 10.1007/s11999-0122749-x). Interview by Seth S. Leopold. Clin Orthop Relat Res. 2013;471:1069-1073.

9. Jaffee KD, Epling JW, Grant W, Ghandour RM, Callendar E. Physician-identified barriers to intimate partner violence screening. J Womens Health (Larchmt). 2005;14:713-720
10. Kyriacou DN, Anglin D, Taliaferro E, Stone S, Tubb T, Linden JA, Muelleman R, Barton E, Kraus JF. Risk factors for injury to women from domestic violence against women. $N$ Engl J Med. 1999;341:1892-1898.

11. Liebschutz JM, Rothman EF. Intimate-partner violence: what physicians can do. N Engl J Med. 2012;367:2071-2073.

12. McCaw B, Berman WH, Syme SL, Hunkeler EF. Beyond screening for domestic violence: a systems model approach in a managed care setting. Am J Prev Med. 2001;21:170-176.

13. Melnick DM, Maio RF, Blow F, Hill EM, Wang SC, Pomerantz R, Kane ML, Graham-Bermann S, Weber J, Farber MS. Prevalence of domestic violence and associated factors among women on a trauma service. J Trauma. 2002;53:33-37.

14. Park ER, Wolfe TJ, Gokhale M, Winickoff JP, Rigotti NA. Perceived preparedness to provide preventive counseling: reports of graduating primary care residents at academic health centers. $J$ Gen Intern Med. 2005:20:386-391.

15. Sprague S, Goslings JC, Hogentoren C, de Milliano S, Simunovic N, Madden K, Bhandari M. Prevalence of intimate partner violence across medical and surgical health care settings: a systematic review. Violence Against Women. 2014;20:118-136.

16. Sprague S, Kaloty R, Madden K, Dosanjh S, Mathews DJ, Bhandari M. Perceptions of intimate partner violence: a cross sectional survey of surgical residents and medical students. J Inj Violence Res. 2013;5:1-10.

17. Sprague S, Madden K, Dosanjh S, Godin K, Goslings JC, Schemitsch EH, Bhandari M. Intimate partner violence and musculoskeletal injury: bridging the knowledge gap in orthopaedic fracture clinics. BMC Musculoskelet Disord. 2013;14:23.

18. Sprague S, Madden K, Simunovic N, Godin K, Pham NK, Bhandari M, Goslings JC. Barriers to screening for intimate partner violence. Women Health. 2012;52:587-605.

19. The Canadian Orthopaedic Association. Intimate partner violence; position statement. Available at: http://www.coa-aco.org/ library/health-policy/intimate-partner-violence.html. Accessed March 24, 2015.

20. Waalen J, Goodwin MM, Spitz AM, Petersen R, Saltzman LE. Screening for intimate partner violence by health care providers: barriers and interventions. Am J Prev Med 2000;19:230-237.

21. Wathen CN, Tanaka M, Catallo C, Lebner AC, Friedman MK, Hanson MD, Freeman C, Jack SM, Jamieson E, Macmillan HL; McMaster IPV Education Research Team. Are clinicians being prepared to care for abused women? A survey of health professional education in Ontario, Canada. BMC Med Educ. 2009;9:34.

22. Wijma B, Schei B, Swahnberg K, Hilden M, Offerdal K, Pikarinen U, Sidenius K, Steingrimsdottir T, Stoum H, Halmesmäki E; Nordic cross-sectional study. Emotional, physical, and sexual abuse in patients visiting gynaecology clinics: a Nordic cross-sectional study. Lancet. 2003;361:2107-2113.

23. World Health Organization. Responding to intimate partner violence and sexual violence against women: WHO clinical and policy guidelines. Available at: http://apps.who.int/iris/bitstream/ 10665/85240/1/9789241548595_eng.pdf. Accessed March 24, 2015. 